\title{
Evolution of cellular structures on oblique detonation surfaces
}

\author{
Honghui Teng ${ }^{1}$, Hoi Dick $\mathrm{Ng}^{2}$, Kang $\mathrm{Li}^{1}$, Changtong Luo ${ }^{1}$, Zonglin Jiang ${ }^{1}$ \\ ${ }^{1}$ State Key Lab of High Temperature Gas Dynamics, Institute of Mechanics, Chinese Academy of Sciences, \\ Beijing 100190, China \\ ${ }^{2}$ Department of Mechanical and Industrial Engineering, Concordia University, Montreal, QC, \\ H3G 1M8, Canada
}

Abstract: In this study, numerical simulations using the inviscid Euler equations with one-step Arrhenius chemistry model are carried out to investigate the effects of activation energy and wedge angle on the stability of oblique detonation surfaces. Two kinds of cellular structure are studied, one is featured by a single group of transverse waves traveling upstream, referred to as LRTW (left-running transverse waves), and the other is featured by additional RRTW (right-running transverse waves). The present computational simulation reveals the formation of un-reacted gas pockets behind the cellular oblique detonation. Numerical smoked foil records are produced to show the emergence of the two types of transverse waves and the evolution of the unstable cellular structure of the oblique detonation. The transverse wave dynamics, including the colliding, emerging and splitting types, are found to be similar to the normal detonation propagation, demonstrating the instability mechanism is originated from the inherent instability of cellular detonations. Statistical analysis on the cellular structure is carried out to observe quantitatively the influences of activation energy and wedge angle. Results from the parametric study show that high activation energy and low wedge angle are favorable to the LRTW formation. However, the condition for the RRTW formation is more complex. In the case of low activation energy, small wedge angle is beneficial to the RRTW formation, as to the LRTW formation. In contrary, for high activation energy, there appears one moderate wedge angle favoring the RRTW formation and giving the shortest length between the onset of both LR and RR transverse waves. For quantitative comparison, we analyze the variation of two distances with the wedge angle, one is between the detonation initiation and LRTW formation points, and the other between LRTW and RRTW formation points. Results show the latter is relatively less pronounced than the former, indicating the RRTW formation depends mainly on the activation energy and the generation of LRTW.

Keywords: detonation, instability, cellular structure, transverse wave, numerical simulations 


\section{Introduction}

The phenomenon of oblique detonation induced by a supersonic combustible flow over an inclined wall has long attracted interest because of its potential application to detonation-based hypersonic propulsion systems for high propulsive efficiency [1,2]. Examples of the application of oblique detonation are the development of continuous detonation wave engines and ram accelerators [3].

In the literature a wealth of research studies on oblique detonations can be found. For instance, the simplified structure in most pioneering analysis is modeled as an oblique shock wave followed by an instantaneous post-shock heat release. Using numerical simulation Li et al. [4] revealed that the multi-dimensional oblique detonation structure consists of a non-reactive oblique shock, an induction region, a set of deflagration waves, and an oblique detonation surface. In this description the oblique shock-oblique detonation transition occurs abruptly giving a $\lambda$-like structure in the vicinity of the leading edge of the wedge. Such structure was verified from experiments [5], and is often considered as the standard model of oblique detonations, e.g., [6,7]. Nevertheless, later studies had revealed other type of transition structures due to various complicated unsteady processes. For example, Vlasenko et al. [8] showed that the shock-to-detonation transition may occur smoothly by an arc shock, rather than occurring in a multi-wave interaction point. A number of parametric studies were carried out numerically to investigate the dependence of the transition type on various initial conditions and flow parameters such as the incoming flow Mach number, wedge angle and the reactivity of the mixture $[7,9,10]$. It is noteworthy that most early numerical studies focused primarily on the stable structure of ODW and the transition from oblique shock to 
detonation, i.e., how the oblique detonation is established. On the other hand, the detailed characteristics of the oblique detonation surface, i.e., how the cellular oblique detonation structures form and evolve, have not yet been fully understood.

Another fundamental characteristic of ODW is the instability of the formed detonation. Indeed from both experiments [5] and numerical simulations [9-14], it was observed that the oblique detonation surface can be unstable with the generation of triple points and fine scale cellular unstable structures very similar to normal cellular detonation. Numerically, it was found by Choi et al. [11] using mixtures of different activation energies that these unstable oblique detonation structures cannot be captured without sufficient numerical resolution. In the numerical results of unstable ODW front obtained by Choi et al. [11], the structure is featured mainly by a single group of transverse waves traveling upstream, referred to as LRTW (left-running transverse waves). This is different from normal detonations with one pair of triple points traveling in opposite direction. By using higher order numerical schemes and extending the computational domain allowing the instability to further develop, recent numerical simulations $[12,13]$ indeed reveals the existence of RRTW (right-running transverse waves) in ODW.

Although these two kinds of cellular structures are observed, i.e., structure featured either by a single-sided triple point or dual-headed triple point, their origin and formation mechanism are still not clear. These characteristics are significant to understand the ODW dynamics and performance of the oblique detonation propulsion system. Recently, Teng et al. [14] demonstrated that the LRTW derives from small perturbation upstream, induced by the interior instability unconditionally. Gui et al. [12] noticed that with variation in activation 
energy of the combustible mixture, the surface disturbance becomes more and more significant, which induces the LRTW and RRTW successively. Verreault et al. [13] observed more closely that the formation of RRTW derives from the compression wave, which may be amplified along the oblique detonation surface. Despite these aforementioned studies and observations by different researchers, the formation of both transverse waves, especially RRTW, has not been studied systematically with different parameters. As for the simulations of normal cellular detonations, the difficulty in investigating this problem is due to the numerical requirement of a wide computational domain covered with a sufficient level of numerical resolution so to reveal correctly the final transverse wave structure of ODW. In this study, the oblique detonations with various activation energies and wedge angles are simulated. To ensure convergence of the numerical results, numerical resolution study is also performed. The instability characteristics of the ODW structure are then analyzed by examining the resolved flow fields. A quantitative study on the transverse waves is also performed by using a statistic method.

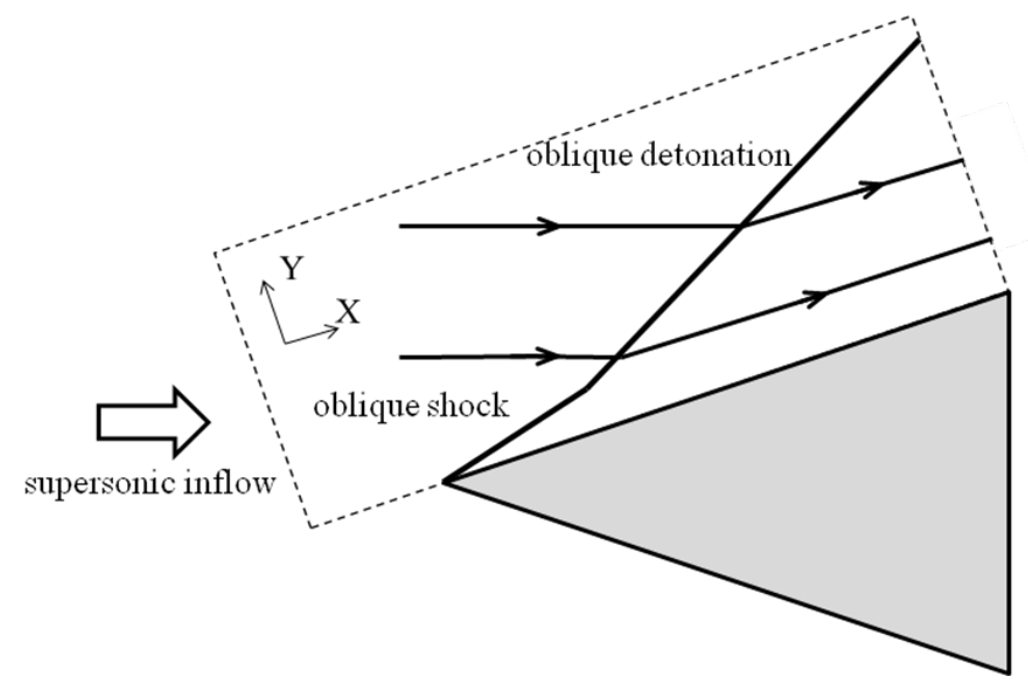

Figure 1. A schematic of the oblique detonation wave induced by the wedge in the combustible gas mixtures 


\section{Numerical methods}

The numerical method follows closely our early work on oblique detonation $[10,14]$. A schematic of the oblique detonation wave induced by the wedge in a combustible gas mixture is shown in Fig. 1. An oblique shock wave forms first at the wedge front, and if the post-shock temperature is sufficiently high, an exothermic chemical reaction begins and induces an oblique detonation. For convenience, the coordinate is rotated to the direction along the wedge surface, and the computational domain is shown in region enclosed by the dashed line. Hence, the Cartesian grid in the rectangular domain is aligned with the wedge surface. Similar to previous numerical studies on oblique detonations [9-15], the present analysis is based on the inviscid, reactive two-dimensional Euler equations. The non-dimensional governing equations with a single-step, irreversible chemical reaction are of the form:

$$
\begin{gathered}
\frac{\partial \rho}{\partial t}+\frac{\partial(\rho u)}{\partial x}+\frac{\partial(\rho v)}{\partial y}=0 \\
\frac{\partial(\rho u)}{\partial t}+\frac{\partial\left(\rho u^{2}+p\right)}{\partial x}+\frac{\partial(\rho u v)}{\partial y}=0 \\
\frac{\partial(\rho v)}{\partial t}+\frac{\partial(\rho u v)}{\partial x}+\frac{\partial\left(\rho v^{2}+p\right)}{\partial y}=0 \\
\frac{\partial \rho e}{\partial t}+\frac{\partial[\rho u(e+p)]}{\partial x}+\frac{\partial[\rho v(e+p)]}{\partial y}=0 \\
\frac{\partial \rho \lambda}{\partial t}+\frac{\partial(\rho u \lambda)}{\partial x}+\frac{\partial(\rho v \lambda)}{\partial y}=\rho \dot{\omega}
\end{gathered}
$$

with 


$$
\begin{gathered}
e=\frac{p}{(\gamma-1) \rho}+\frac{1}{2}\left(u^{2}+v^{2}\right)-\lambda q \\
p=\rho T \\
\dot{\omega}=k(1-\lambda) \exp \left(-\frac{E_{a}}{T}\right)
\end{gathered}
$$

where $\rho, u, v, p, e$ denote the density, velocity in $x$ - and $y$-direction, pressure and the total energy, respectively. All the flow variables have been made dimensionless by reference to the uniform unburned state (with subscript o). For the chemical reaction, $\lambda$ is the reaction progress variable which varies between 0 (for unburned reactant) and 1 (for product), and $q$ is the amount of heat release non-dimensionalized by $R T_{0}$. The reaction is controlled by the activation energy $E_{\mathrm{a}}$ (also non-dimensionalized by $R T_{\mathrm{o}}$ ) and the pre-exponential factor $k$. The latter is chosen to define the spatial and temporal scales. The mixture is assumed to be ideal and calorically perfect. In this study, we fix the dimensionless parameters with the values $q=$ 50 and $\gamma=1.2$. The governing equations are discretized on Cartesian uniform grids and solved with the MUSCL-Hancock scheme with Strang's splitting. The MUSCL-Hancock scheme is formally a second-order extension to the Godunov's first order upwind method by constructing the Riemann problem on the inter-cell boundary [16]. The scheme is made total variation diminishing (TVD) with the use of slope limiter MINBEE, and the HLLC solver is used for the Riemann problem. If not specified, a numerical resolution of 32 points per half reaction length $l_{1 / 2}$ of a CJ detonation is used in all simulation cases. This equivalently provides approximately $10 \mathrm{pts} / \mathrm{l}_{1 / 2}$ of an overdriven ODW (along its normal direction) in flow conditions considered in this work. 


\section{Results and discussion}

\subsection{Cellular structures and the effect of activation energy}

In this study, the inflow Mach number is fixed to 12.0 and different wedge angles are considered. The activation energy $E_{\mathrm{a}}$ is the main bifurcation parameter as considered in most studies of detonation instability, see [17-22]. Here, the activation energy varies between 27.0 and 33.0. Inflow conditions are fixed at the free-stream values in both the left and upper boundaries of the domain. Outflow conditions extrapolated from the interior are implemented on the right and lower boundaries before the wedge. Slip boundary conditions are used on the wedge surface, which starts from $x=0.5$ on the lower boundary. Initially the whole flow field has uniform density, velocity, and pressure, which are calculated according to wedge angle $\theta$. As provided earlier, a resolution of 32 grids per $l_{1 / 2}$ has been used in this simulation, and further discussion on the resolution requirement will be provided in later section.

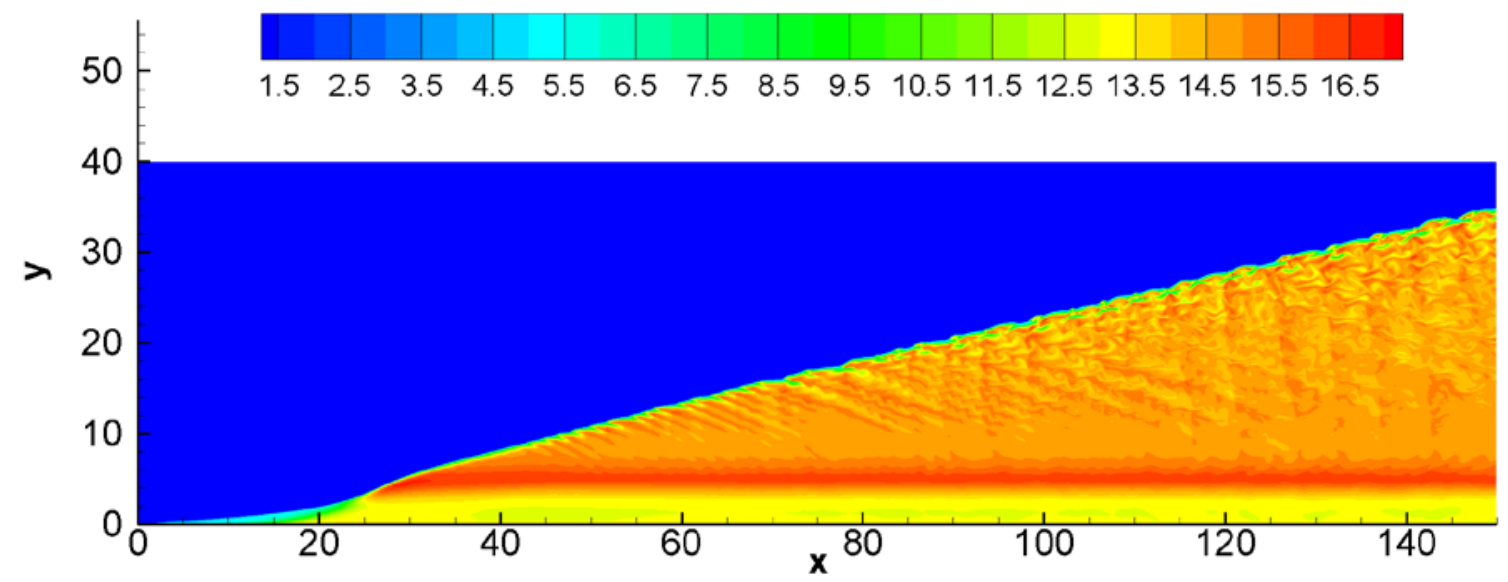

Figure 2. Temperature flow fields of oblique detonations with $E_{\mathrm{a}}=31.0$ and $\theta=26^{\circ}$. 

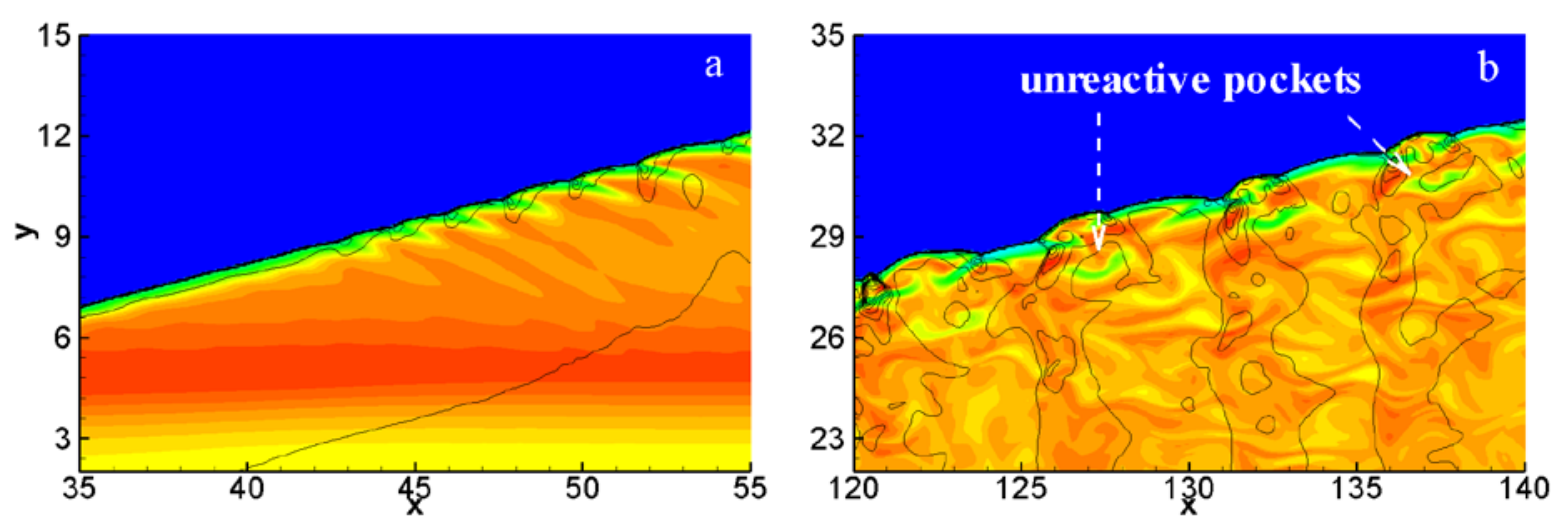

Figure 3. Temperature and pressure (black lines) of oblique detonations with $E_{\mathrm{a}}=31.0$ and $\theta$ $=26^{\circ}$. (a) first kind of cellular structure; (b) second kind of cellular structure.

Figure 2 shows the temperature contours of oblique detonation waves with the activation energy $E_{\mathrm{a}}=31.0$ and wedge angle $\theta=26^{\circ}$. An oblique shock forms first, and then an oblique detonation initiates around $x=25$. Due to the heat release, the oblique detonation has a higher deflection angle than the oblique shock. After its initiation, the detonation surface is initially stable, but instability begins to manifest soon downstream. An unstable structure forms on the surface eventually, and we can observe two kinds of cellular structure. Figure 3 shows the fine details on different positions of the unstable surface. The first one is characterized by only the LRTW, which are convected downstream in the laboratory coordinate. The flame brush with sharp tips appears behind the unstable surface, as shown in Fig. 3a. The other kind of structure is characterized by both LRTW and RRTW, as shown in Fig. 3b. From the pressure contours, it can be found that RRTW remains weaker than LRTW, but the appearance of RRTW changes the oblique detonation significantly. Although similar results of cellular oblique detonations have been simulated, the fine structures, such as the un-reacted pocket revealed in Fig. 3b, have never been reported possibly due to the low resolution and low degree of instability (i.e., high overdrive or low activation energy) used in previous works. 


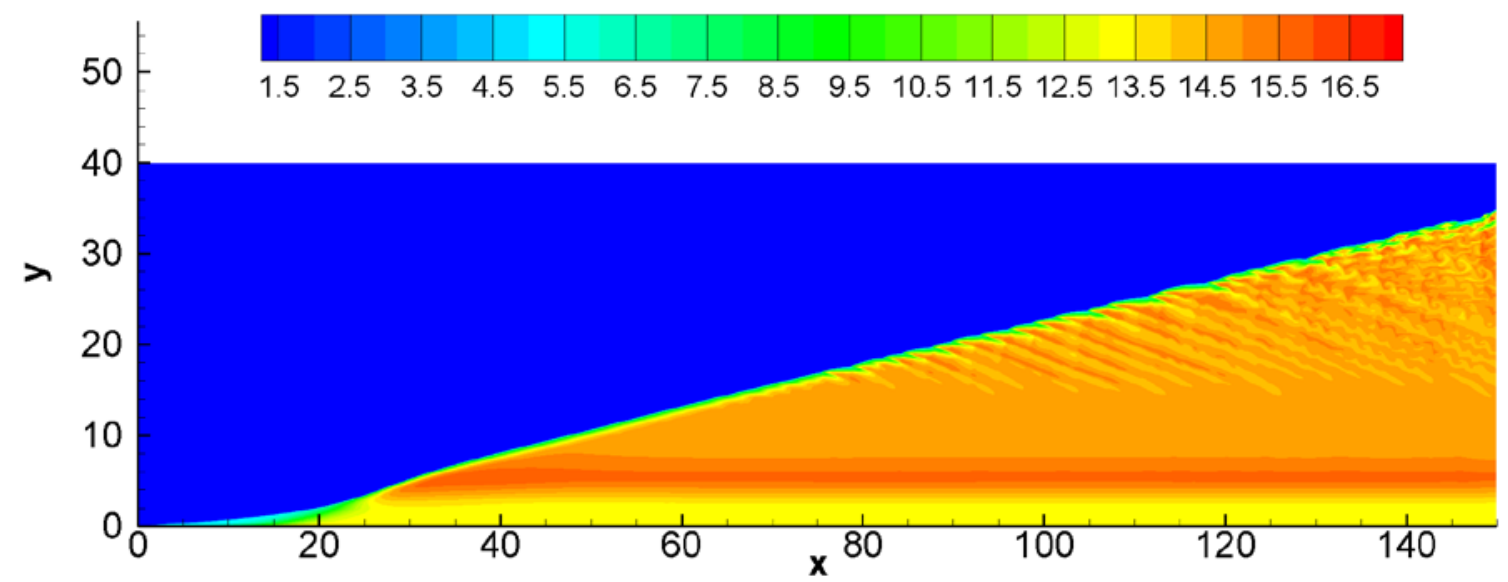

Figure 4. Temperature flow fields of oblique detonations with $E_{\mathrm{a}}=27.0$ and $\theta=26^{\circ}$.

To study the effect of activation energy, oblique detonations are simulated with the same inflow Mach number and wedge angle with different value of $E_{\mathrm{a}}$. Pre-exponential factor $k$ is adjusted according to the activation energy value, so to make the half reaction length $l_{1 / 2}$ unity. Figure 4 shows the temperature contours of the oblique detonation wave with activation energy $E_{\mathrm{a}}=27.0$. Comparing with the last case, the onset of the oblique detonation occurs at approximately the same location. However, the positions where LRTW and RRTW appear move downstream significantly, result which is similar to the previous study by Choi et al. [11]. However, only the effect of activation energy on the LRTW formation has been noticed in previous studies, and the RRTW formation has not been discussed. Moreover, different resolutions are used previously in different studies. To this end, studies with unified high resolution simulation results are necessary to understand the instability mechanism.

Previous results $[13,15]$ demonstrate that high overdrive degree, resulted from an increase of wedge angle or inflow Mach number, can suppress the instability of oblique detonations, and that the oblique detonation instability is equivalent to 1-D overdriven 
detonation instability if they have the same overdrive degree. Our recent results support the fact that high overdrive degree indeed suppresses the instability, but results also show that oblique detonations can remain unstable even with very high overdrive degrees above the neutral stability boundary of 1D normal detonation [14]. In this study, the overdrive degree of the case with $E_{\mathrm{a}}=31.0$ and wedge angle $\theta=26^{\circ}$ is about 1.5 , and the corresponding 1-D normal detonations are stable with activation energies from 27.0 to 31.0. In contrast, we can clearly observe that oblique detonations become unstable and form the cellular structures. Therefore, the neutral stability boundary of 1-D pulsating detonation is not sufficient to assess the stability characteristics of unstable oblique detonations. Since oblique detonation wave is multi-dimensional, the stability characteristic should be better described by the two-dimensional stability analysis of normal cellular detonations [17]. As per discussed in [14], increases in detonation overdrive $f$ even beyond the one-dimensional longitudinal stability boundary, cannot guarantee stability of the steady detonation wave to transverse linear disturbances, which can be excited to originate the detonation cell formation 


\subsection{Resolution study}
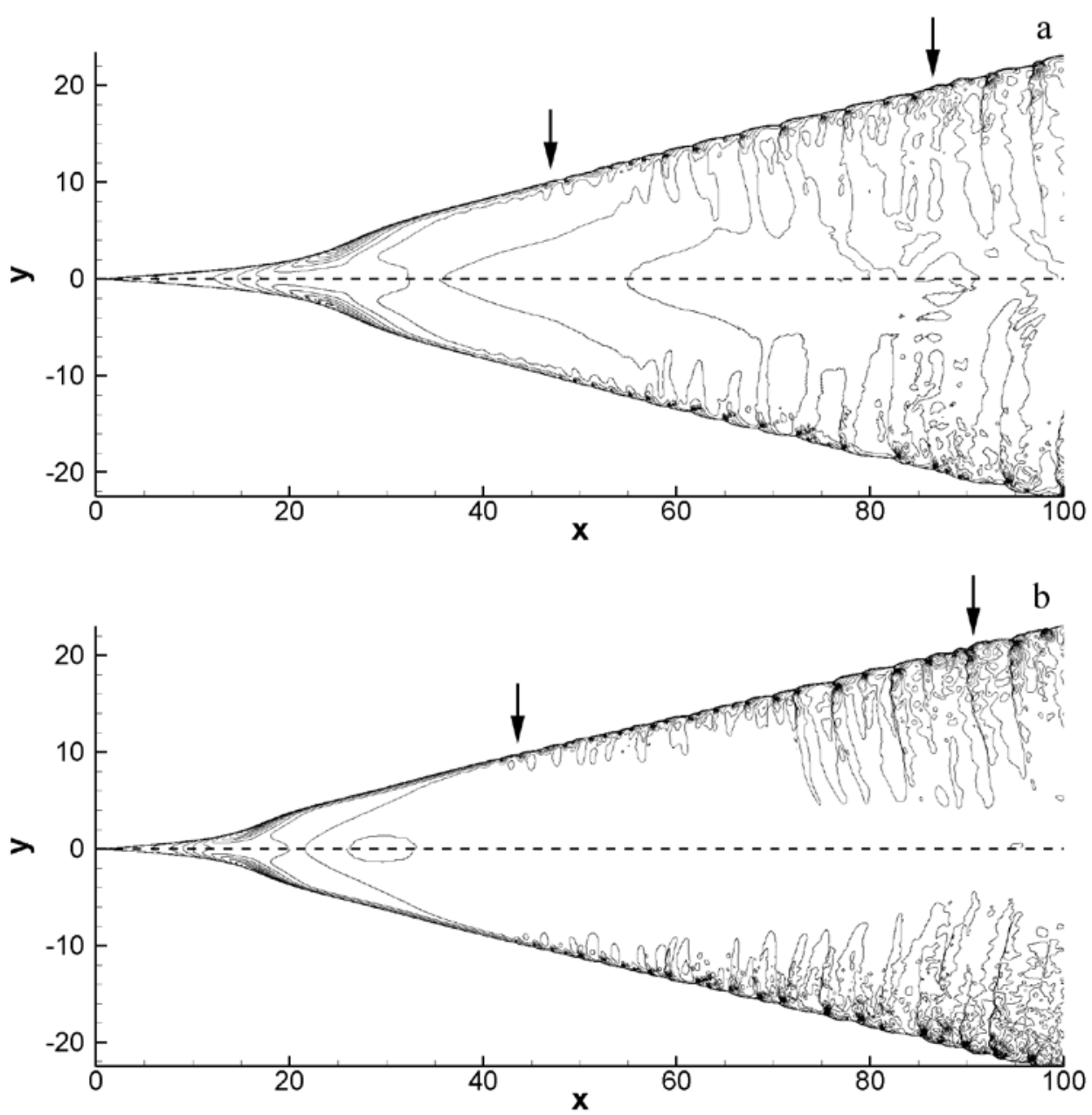

Figure 5. Pressure contours with 32 (upper) and 64 (lower) grid points per half reaction length with (a) $E_{\mathrm{a}}=31.0$ and $\theta=26^{\circ}$; and (b) $E_{\mathrm{a}}=33.0$ and $\theta=28^{\circ}$.

In order to clarify the effect of numerical grid resolution, detonation simulations with different grid sizes are carried out using the same initial and mixture conditions. Figure 5 compares the pressure contours with 32 and 64 grid points per $l_{1 / 2}$ of a CJ detonation. Two test cases are displayed, one is the case with $E_{a}=31.0$ and $\theta=26^{\circ}$, whose flow field has been shown in Fig. 2 and 3, and the other is the case with $E_{a}=33.0$ and $\theta=28^{\circ}$, corresponding to the highest activation energy and wedge angle considered in this study. It is found that the pressure profiles are almost the same in the oblique shock surface and near the wedge, except 
the region of unstable oblique detonation surface. Because the cellular structure evolves from random disturbances, it is impossible to get the exact same flow fields on the detonation surface. To our study on oblique detonation unstable surface, two key positions are critical. One is the position where the surface becomes unstable, i.e., where LRTW forms; and the other is the transition position of double-headed cellular structure, i.e., the location for the appearance of RRTW. With a very coarse computational grid, the surface is planar and remains stable. Increasing the resolution is required to capture the evolution of the unstable ODW surface. With the appearance of the unstable surface using sufficient grid points, the aforementioned key positions continue to move upstream by increasing the resolution up to $32 \mathrm{pts} / \mathrm{l}_{1 / 2}$. However, when the resolution increases from 32 to $64 \mathrm{pts} / \mathrm{l}_{1 / 2}$, these two key positions are found to remain in close vicinity as indicated by the markers in Fig. 5. Due to the non-stationary nature of the flow field, the quantitative estimation of these positions will be discussed in later section using a statistical method. This consistency is important to ensure the instability feature is not sensitive to the numerical resolution. Without enough grid resolution, effect of numerical diffusion can delay the onset of instability, similar to one-dimensional unstable detonations [23]. It is nevertheless found that an equivalent resolution of $32 \mathrm{pts} / \mathrm{l}_{1 / 2}$ is sufficient for the mixture and flow conditions used in the present study of unstable oblique detonation and hence, this resolution is used in all later simulations.

Results using this same degree of resolution have also been reported in recent normal detonation studies $[24,25]$. Oblique detonations are overdriven, so on the direction vertical to the surface, there are fewer grids in half reaction length. However, previous studies show the resolution requirement in oblique detonation seems not as strict as in usual 2-D normal 
cellular detonation. For example, Choi et al. [11] uses equivalently a grid resolution of less 10 $\mathrm{pts} / \mathrm{l}_{1 / 2}$ of an overdriven ODW, and $13 \mathrm{pts} / \mathrm{l}_{1 / 2}$ is used in the recent work by Verreault et al. [13]. Our previous study [14] found that approximately $10 \mathrm{pts} / \mathrm{l}_{1 / 2}$ of an overdriven ODW can reproduce qualitative good results. (Note that this resolution requirement is indeed satisfied in the present work using $32 \mathrm{pts} / \mathrm{l}_{1 / 2}$ of a CJ detonation). This probably derives from the flow characteristics of oblique detonations. The flow is compressed by the oblique detonation, and the post-shock velocity is along the positive x-direction, paralleled with the wedge. Thus, the angle of the flow and surface increases the real grids where fluid experiences, which means the nonlinear instability evolution is not suppressed by relative few grids in half reaction length. This provides an explanation on why the oblique detonation needs fewer grids in half reaction length, although more detailed studies are necessary undoubtedly.

\subsection{Transition of the two kinds of cellular structure}
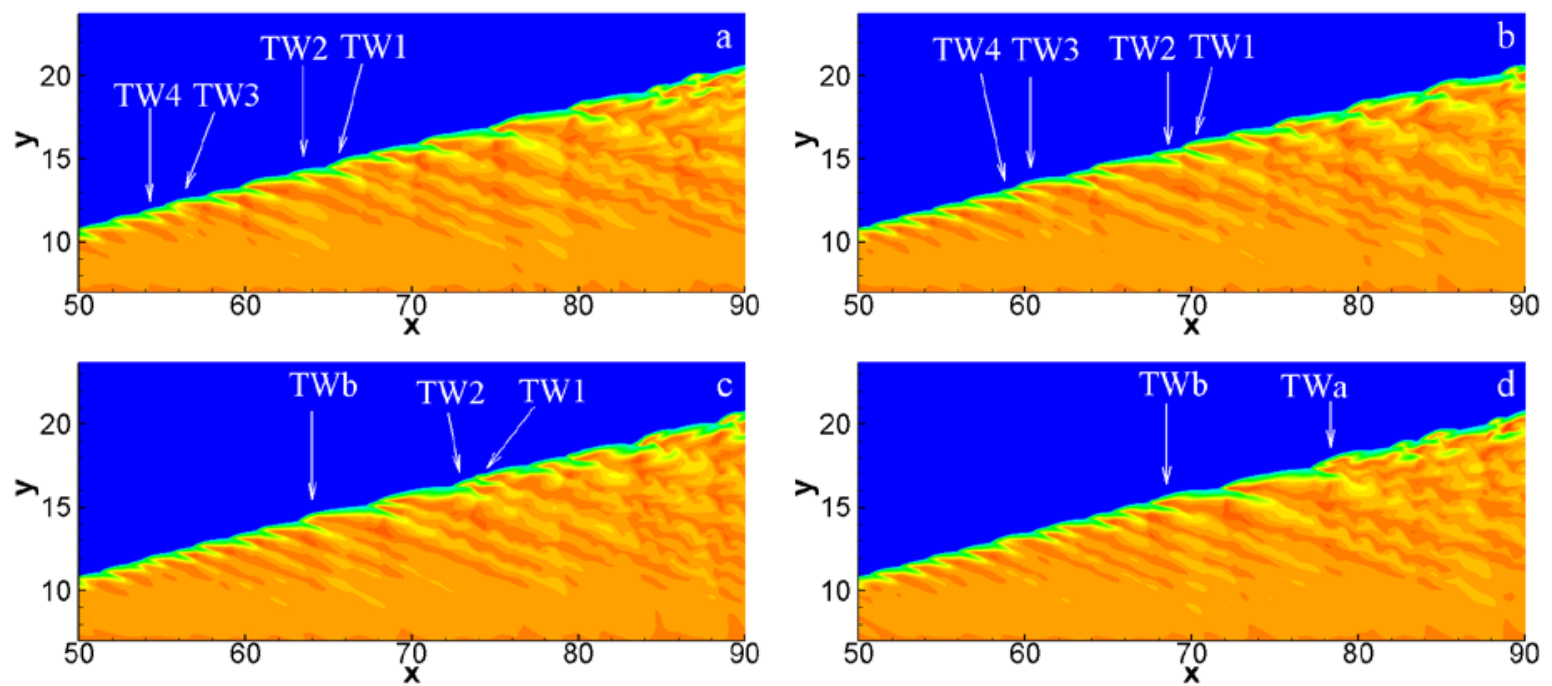

Figure 6. Cellular surface dynamics of oblique detonations with $E_{\mathrm{a}}=31.0$ and $\theta=26^{\circ}$.

In order to further understand the transition mechanism of the two cellular structures, 
dynamic processes of unstable surfaces near the transition point are illustrated in Fig. 6. Initial non-dimensional time corresponds to about 19.0, and the time interval is about 0.72 . In Fig. 6a, four transverse waves are marked with "TW1" to "TW4", and their intervals with each other are close. Following the transverse wave movement, one can observe that the interval between TW1 and TW2 becomes smaller, forming TWa eventually. Similar phenomenon can be observed for TW3 and TW4, forming TWb as shown in Fig. 6c. Newly formed transverse waves have larger interval than that of initial transverse waves. This is crucial for the formation of RRTW, because small disturbance can be amplified behind them, as shown in Fig. 6d.

Numerical smoked foil record using maximum pressure trace is produced and shown in Fig. 7. For the case with activation energy $E_{\mathrm{a}}=31.0$ and wedge angle $\theta=26^{\circ}$ given in Fig. $7 \mathrm{a}$, three regions can be observed on the oblique detonation surface after the initiation. The first region corresponds the planar surface, which is short and locates around $y=20$. The second region where only LRTW exists, shows the trajectory of one-set of triple points. Initially a large number of triple points are generated close to the initiation point of the ODW, but these collide with each other and merge together, as shown in Fig. 7a. The average interval of transverse waves becomes eventually larger in the region between $y=40$ and $y=50$. Subsequently, the emergence of RRTW can be observed, inducing a cellular structure like the normal cellular detonation. The evolution of cellular structures for the case with activation energy $E_{\mathrm{a}}=27.0$ and wedge angle $\theta=26^{\circ}$ is also observed in the numerical smoke foil record shown in Fig. 7b. This case has longer planar surface from the initiation point of the detonation and the RRTW formation also moves downstream. We can observe that some 
triple point tracks merge together and subsequently the average interval becomes larger, similar to the observation in the case with activation energy $E_{\mathrm{a}}=31.0$.
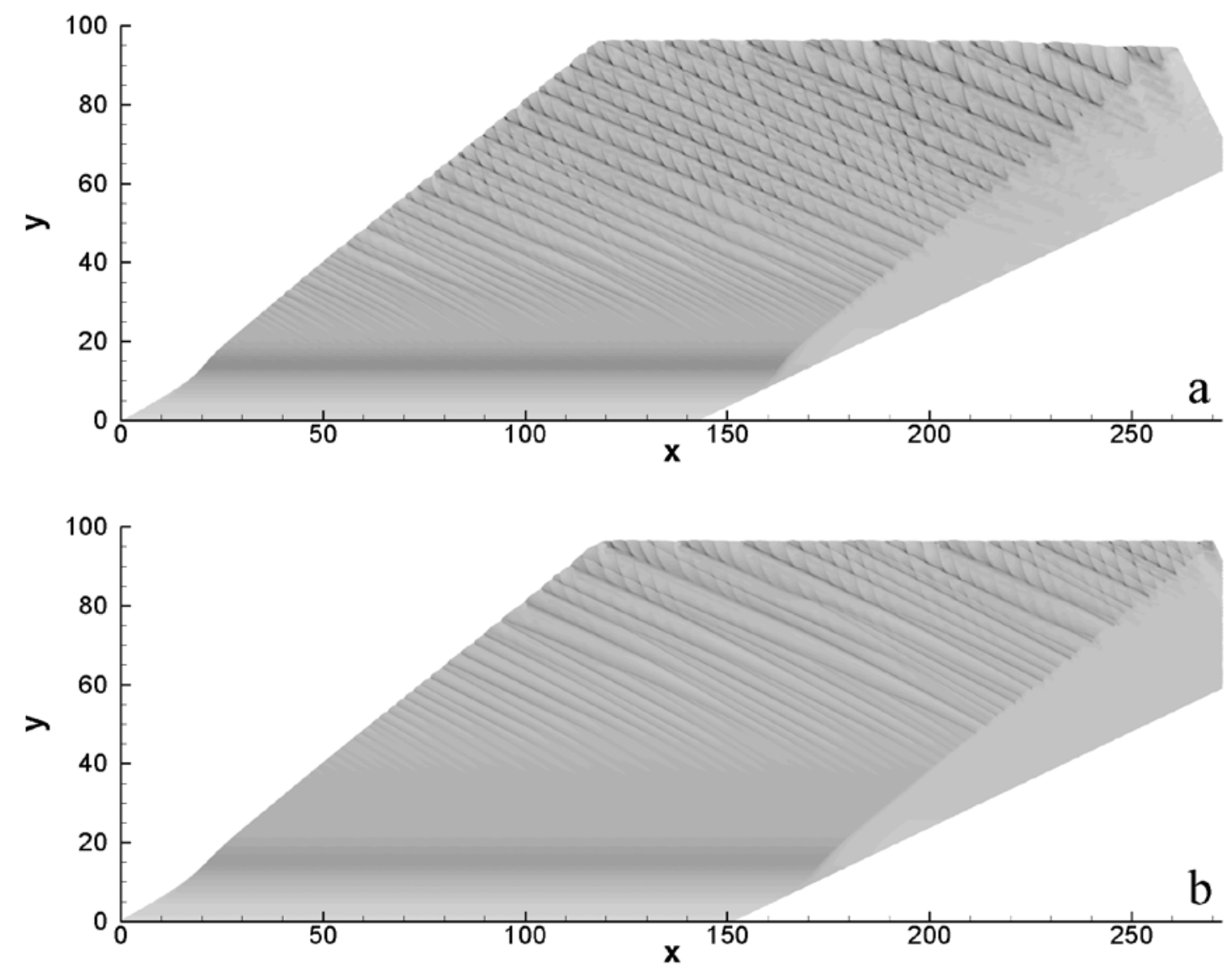

Figure 7. Numerical smoke foil record of cellular oblique detonations with $\theta=26^{\circ}, E_{\mathrm{a}}=$ 31.0(a) and $E_{\mathrm{a}}=27.0(\mathrm{~b})$.

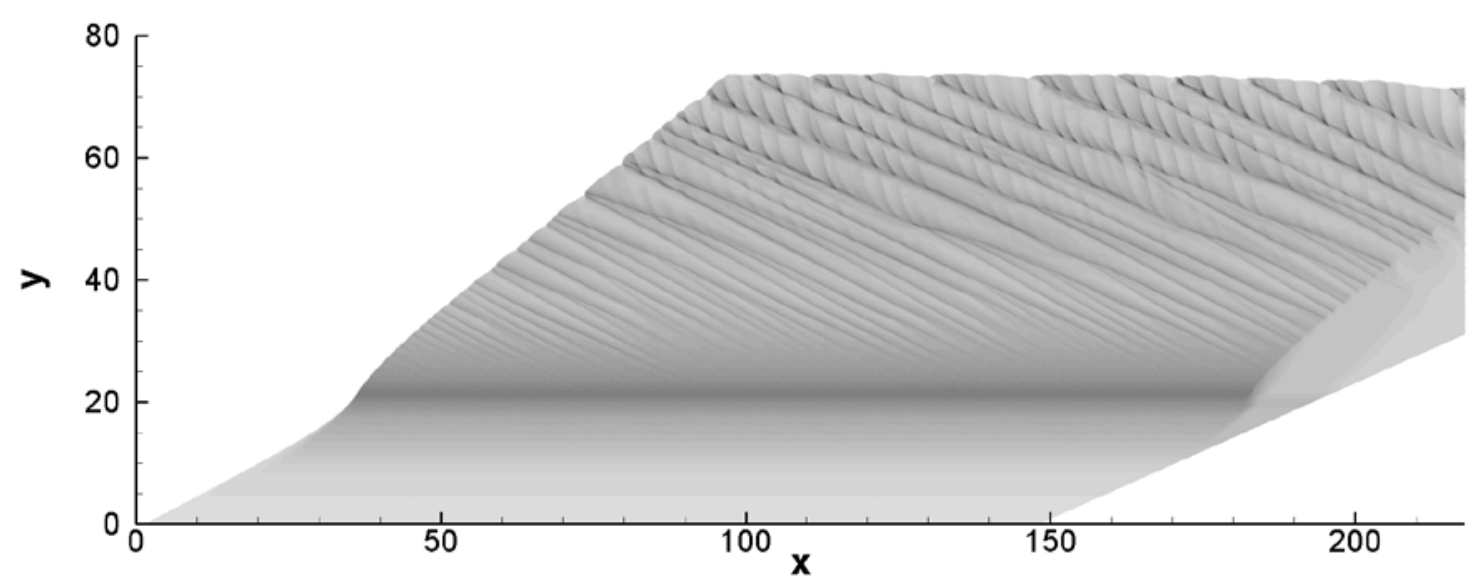

Figure 8. Numerical smoke foil record of cellular oblique detonations with $\theta=24^{\circ}, E_{\mathrm{a}}=$ 33.0. 
Figure 8 shows the numerical smoked foil record for the case with activation energy $E_{\mathrm{a}}=$ 33.0 and wedge angle $\theta=24^{\circ}$. Initiation point of the detonation moves downstream due to the high activation energy of the mixture, and the generation of triple points occur earlier so the planar surface becomes short. Triple point trajectory is similar to that shown in Fig. 7, but some qualitative difference can still be seen. The cell width in this cellular structure changes in a wider spectrum. The large cell width ranges from twice or more than the narrow one. Although the width in Fig. 7 also changes, the difference is not as significant. This supports the fact that the increase of activation energy and decrease of the wedge angle make the oblique detonation more unstable with an irregular cellular structure.

The surface evolution of oblique detonations appears to be similar to that for normal detonations $[24,26,27,28,29,30]$. With the ZND structure as an initial condition, a spectrum of instability modes induces many weak transverse waves on the detonation surface initially. Subsequently the dominant nonlinear instability plays the main role and the merging of weak transverse waves can be observed. When the distance becomes too large between adjacent transverse waves, new transverse waves are easy to form, especially in the case with high activation energy. This phenomenon is also observed in diverging detonations whose surface becomes larger and larger, related with the curvature effects [28,31]. Generally, the merging and splitting of transverse waves appear to be equivalent to those observed in normal detonations, which thus demonstrate that the transition mechanism is due to the inherent instability of detonations. 


\subsection{Statistic analysis on the oblique cellular detonation structure}

To provide a quantitative description of the transition, two key points, KP1 and KP2, are defined. KP1 corresponds to the location where LRTW first emerges, and KP2 corresponds to the one where RRTW emerges. Due to the non-stationary nature of the flow field, these points always move and as a result, their locations are difficult to define. Recently, Sharpe and Radulescu [32] proposed a statistic method to deal with irregular detonation cells, whose idea is used here. First, the long time computation is performed after the oblique detonation forms, and the instantaneous location of these key points are saved every several steps. If the total steps are large enough, significant amounts of instantaneous key points can be recorded. The probability of KP1 and KP2 on certain position can then be calculated through post-processing, which is independent on the non-stationary flow. Sharpe \& Radulescu's method eliminates the uncertainty induced by the instability, and it is indeed found that the probability profiles are almost invariable if the obtained number of instantaneous key points is large enough.

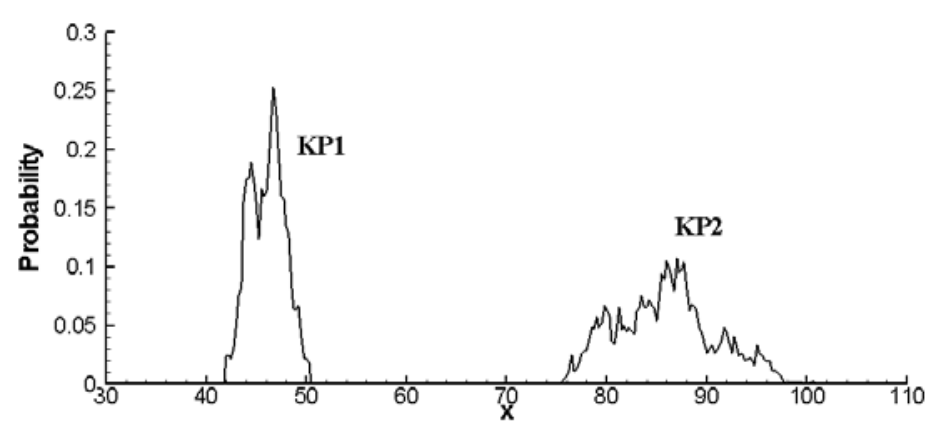

Figure 9. Probability distributions of KP1 and KP2 with $E_{\mathrm{a}}=31.0$ and $\theta=26^{\circ}$. 
An example of probability distributions of KP1 and KP2 are shown in Fig. 9. KP1 moves in a narrow range around 40-50, while KP2 moves in a wide range around 75-100. We use $50 \%$ probability to indicate the position of these key points. For KP1 and KP2 shown in Fig. 9, the position is 46.4 and 87.2 , respectively. It should be noticed that this probability is influenced by the type of detector used in both KP1 and KP2. Regardless of the type, these detectors both work along the line paralleled with $y$-axis. KP1 detector uses the reaction progress variable $\lambda$, which is equal to 1 near the wedge while 0 before the oblique surface. Along the line on the positive $y$-axis direction, $\lambda$ decreases monotonously for planar surface, while for cellular surface, $\lambda$ re-increases at certain positions. The first re-increasing position from the left is detected and marked by KP1. To ensure the re-increasing is real and not induced by the numerical error, the threshold $5 \%$ is used, meaning the value at certain grid must be $5 \%$ higher than its neighboring-grid value is necessary. The $5 \%$ criterion may be changed to $10 \%$ or $15 \%$, which will make the position move downstream to 46.9 or 47.5 .

The type of KP2 detector is difficult to choose due to a more complicated flow field. Both the reaction progress variable and the pressure/density are not suitable to detect the RRTW formation. In this study, the detector we use is the $x$-direction velocity with a threshold. Usually the inflow velocity is the maximum one in the whole flow field due to oblique shock/detonation compression, but after the RRTW forms, new maximum velocity appears benefiting from the RRTW's shock acceleration. Hence, KP2 detector is designed to compare the post-shock velocity with the inflow velocity. At certain instant, KP2 first 
searches the maximum $x$-component of velocity on the detonation surface along the line paralleled with the $y$-axis; the value is then compared with the pre-shock velocity in the same $x$-direction. If the maximum velocity is $5 \%$ higher than the pre-shock velocity, RRTW is thought to appear. The first position of RRTW formation from upstream is therefore marked by KP2. Different from the KP1 detector, the threshold has a significant effect on the probability distribution. Replacing $5 \%$ with $10 \%$ or $15 \%$, the position moves downstream from 87.2 to 93.8 or 104.5. This demonstrates that the RRTW formation is more sensitive to the chosen type of detector. To exclude the uncertainty of different cases, the threshold 1.05 is used for the detector of KP2 in all cases. KP1 and KP2 are used to define the characteristic lengths $L_{1}$ and $L_{2}$, which stand for the length of the planar surface and the length of the first kind of cellular structure, respectively. $L_{1}$ starts at the detonation initiation point and terminates at KP1, while $L_{2}$ starts at KP1 and ends at KP2. Their dependences on the wedge angle and activation energy are important parameters of oblique detonation surface instability.

A parametric study using four group cases with activation energies $E_{\mathrm{a}}=27.0,29.0,31.0$ and 33.0 are carried out, and for each activation energy five cases with wedge angles $\theta=24^{\circ}$ to $28^{\circ}$ are simulated. Figure 10 shows the length of planar surface $L_{1}$, the length of first kind of cellular structure $L_{2}$, and the total length of $L_{1}+L_{2}$. For the length $L_{1}$ shown in Fig. 10(a), it becomes longer with the increase of activation energy. For mixtures with low activation energy, the unstable surface takes time to develop due to a low instability growth rate. On another note, it is found that length $L_{1}$ generally increases when the wedge angle increases. These results are in agreement with previous studies $[13,15]$. However, here it is found that 
the dependence of $L_{1}$ on the wedge angle is strongly influenced by the activation energy, which is not clarified before. In the cases of activation energy $E_{a}=27.0$ and 29.0, dramatic increases of $L_{1}$ appears between wedge angle $\theta=25^{\circ}$ to $27^{\circ}$. Therefore, significant $L_{1}$ difference can be observed in the case of wedge angle $\theta=28^{\circ}$, compared to the case of low wedge angle $\theta=24^{\circ}$.
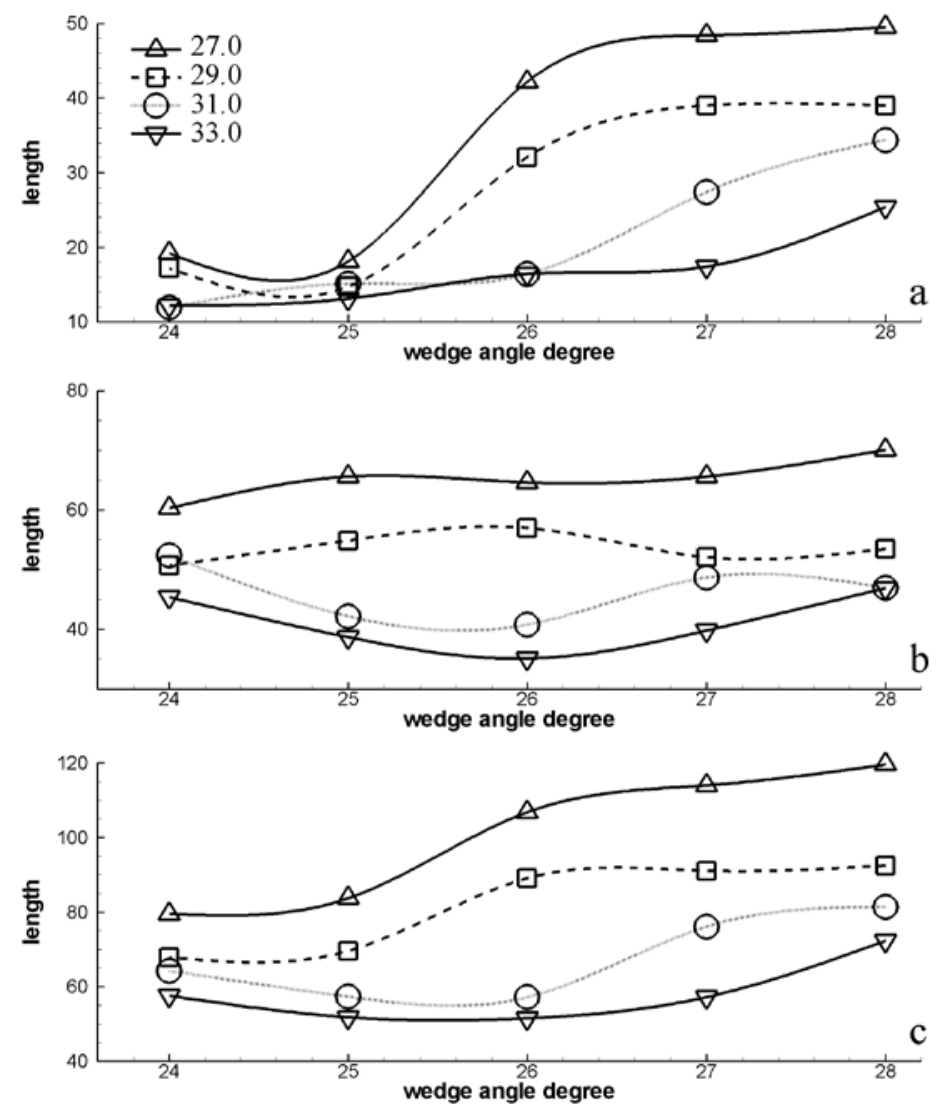

Figure 10. Characteristic length of oblique detonation surface: (a) length of planar surface $L_{1}$; (b) length of first kind of cellular structure $L_{2}$; (c) total length $L_{1}+L_{2}$

In contrary, it is observed that the variation of length $L_{2}$ is different from that of $L_{1}$, as shown in Fig. 10(b). In the cases of activation energy $E_{a}=27.0, L_{2}$ increases when the wedge angle increases, similar to the corresponding $L_{1}$ variation. However, for activation energy $E_{a}$ $=29.0, L_{2}$ increases when wedge angle changes from $24^{\circ}$ to $26^{\circ}$, and then decreases slightly when wedge angle changes from $26^{\circ}$ to $28^{\circ}$. The value $L_{2}$ of wedge angle $\theta=28^{\circ}$ is almost 
the same as $L_{2}$ of wedge angle $\theta=24^{\circ}$. In the cases of activation energy $E_{a}=31.0, L_{2}$ decreases first and then increases. The $L_{2}$ value of wedge angle $\theta=28^{\circ}$ is slightly lower than $L_{2}$ of wedge angle $\theta=24^{\circ}$. Similar phenomenon can be observed in the cases of activation energy 33.0. Hence, the $L_{2}$ dependence on the wedge angle is found to be more complex. $L_{1}$ variations show quantitative differences in the cases of activation energies, but $L_{2}$ variation show qualitatively different trends. Nevertheless, the magnitude of $L_{2}$ variation with wedge angle appears to be relatively less as compared to $L_{1}$ dependence and that $L_{2}$ is more affected mainly by activation energy.

Total length $L_{1}+L_{2}$ denotes the length of transient pattern on oblique detonation surface, shown in Fig. 10(c). In all cases, the total length of wedge angle $\theta=28^{\circ}$ is higher than that of wedge angle $\theta=24^{\circ}$, demonstrating the overall effects are decided by $L_{1}$. However, the influence of $L_{2}$ is more obvious in the cases of activation energy $E_{\mathrm{a}}=31.0$ and 33.0, whose total length has a trough near the wedge angle $\theta=26^{\circ}$. In such case, the chemical effect due to high mixture sensitivity thus dominates the stability effect from the overdriven flow characteristics.

In short, $L_{1}$ and $L_{2}$ are defined to describe the instability of oblique detonation unstable surfaces in this study. Alternatively, these stand for the degree of difficulty to form LRTW and RRTW. Previous studies found that the activation energy and wedge angle (or overdrive degree) have major effect on the instability. This study demonstrates that high activation energy and small angle (low overdrive) are beneficial to the LRTW formation. However, more complex trends are observed in the RRTW formation, and different variations exist depending on the range of activation energy. For high activation energy above 30.0, $L_{2}$ 
variation is not monotonic, and the formation of RRTW is most favorable for the case with modest wedge angle. Nevertheless, compared to $L_{1}$ variation, the dependence of $L_{2}$ on wedge angle is less significant and it appears that the RRTW formation relies more on the activation energy and the first emergence of LRTW. The underlying mechanism of these phenomena is still not clear due to the complicated interaction of shock and combustion on the unstable surface. More work is thus necessary to analyze these parametric results obtained in this study.

\section{Conclusion}

Oblique detonations are simulated to study the formation of the unstable cellular detonation surface. Similar to previous studies, two kinds of cellular structure are observed. One is featured by a single group of transverse waves traveling upstream, and the other is featured by additional RRTW. Benefiting from high resolution simulation, un-reacted pocket is observed on the oblique detonation surface for the first time. In order to obtain qualitatively convergence of the results, 32 grids in half reaction length are used and double-grid resolution test is carried out for verification.

Based on the results of the present numerical simulation, the cellular structure evolution is examined to shed light on the instability mechanism. The numerical smoked foil records are produced to display triple-point traces and elucidate the different regimes of the cellular structure evolution. The transverse wave dynamics, including colliding, emerging and splitting, are identified. These are found to be similar to the normal detonation propagation, demonstrating the instability mechanism is the inherent instability of cellular detonations. 
Statistical analysis on cellular structure is carried out to study the effects of activation energy and wedge angle. The present parametric results indicate that high activation energy and low wedge angle are beneficial to the LRTW formation, but RRTW formation is complicated. In the cases of low activation energy, small wedge angle is relatively more favorable to the RRTW formation, like the LRTW formation; in the case of high activation energy, there exists one moderate wedge angle, corresponding to the shortest length of the first cellular structure. Nevertheless, the variation of $L_{2}$, i.e., the distance between the formation of LRTW and RRTW, appears to be less dependent on the wedge angle and the overall results suggests that the RRTW formation is more affected by the activation energy and the existence of LRTW. Due to the complicated interaction of shock and combustion, the inherent mechanism leading to these phenomena remains unclear and hence, more research effort is necessary to investigate this problem of unstable cellular oblique detonation in the future.

\section{Acknowledgment}

The research is supported by The National Natural Science Foundation of China NSFC

No.11372333 and 51376165; and the Natural Sciences and Engineering Research Council of Canada (NSERC).

\section{References}

1 K. Kailasanath, AIAA J. 41 (2003) 145-159.

2 M.A. Nettleton, Shock Waves 10 ( 2000) 9-22.

3 P. Wolanski, Proc. Combust. Inst. 34(1) (2013) 125-158.

4 C. Li, K. Kailasanath, E.S. Oran, Phys. Fluids 6 (1994) 1600-1611.

5 C. Viguier, L. Figueira da Silva, D. Desbordes, B. Deshaies, Proc. Combust. Inst. 26 (1996) 3023-3031.

6 G. Fusina, J. P. Sislian, B. Parent, AIAA J. 43 (2005) 1591-1604.

7 J. Y. Choi, E. J., Shin, I. S. Jeung, Proc. Combust. Inst. 32 (2009) 2387-2396.

8 V. V. Vlasenko, V.A. Sabel'nikov, Combust. Explos. Shock Waves 31 (1995) 376-389. 
9 M. V. Papalexandris, Combust. Flame 120 (2000) 526-538.

10 H. H. Teng, Z. L. Jiang, J. Fluid Mech. 713 (2012) 659-669.

11 J. Y. Choi, D. W. Kim, I. S. Jeung, F. Ma, V. Yang, Proc. Combust. Inst. 31 (2007) 2473-2480.

12 M. Y. Gui, B. C. Fan, G. Dong, Acta Mech. Sin. 27 (2011) 922-928.

13 J. Verreault, A. J. Higgins, R. A. Stowe, Proc. Combust. Inst. 34 (2013) 1913-1920.

14 H. H. Teng, Z. L. Jiang, H. D. Ng, J. Fluid Mech. 744 (2014) 111-128.

15 M. J. Grismer, J. M. Powers, Shock Waves 6 (1996) 147-156.

16 E. F. Toro, Riemann solvers and numerical methods for fluid dynamics, Second ed., Springer, Berlin, 1999.

17 M. Short, D.S. Stewart, J. Fluid Mech. 368 (1998) 229-262.

18 G. J. Sharpe, J. J. Quirk, Combust. Theory Model. 12 (2008) 1-21.

19 D. S. Stewart, A. R. Kasimov, J. Prop. Power 22 (6) (2006) 1230-1244.

20 J. M. Powers, J. Prop. Power 22(6) (2006) 1217-1229

21 H. Ng, A. Higgins, C. Kiyanda, M. Radulescu, J. Lee, K. Bates, N. Nikiforakis, Combust. Theory Model. 9 (2005) 159-170.

22 H.D. Ng, F. Zhang, Detonation instability. In Shock Wave Science and Technology Reference Library, Vol. 6 (ed. Zhang, F.), (2012) pp. 107-212. Springer.

23 C. M.Romick, T. D. Aslam, J. M. Powers, J. Fluid Mech. 699 (2012) 453-464.

24 B. S. Taylor, A. R. Kasimov, D. S. Stewart, Combust. Theory Model. 13 (2009) 973-992.

25 G. J. Sharpe, J. J. Quirk, Combust. Theory Model. 12 (2008) 1-21.

26 V. N. Gamezo, D. Desbordes, E. S. Oran, Combust. Flame 116 (1999) 154-165.

27 M. I. Radulescu, G. J. Sharpe, C. K. Law, J. H.S. Lee, J. Fluid Mech. 580 (2007) 31-81.

28 Z. Jiang, G. Han, C. Wang, F, Zhang, Combust. Flame 156 (2009) 1653-1661.

29 K. Mazaheri, Y. Mahmoudi, M. I. Radulescu, Combust. Flame 159 (2012) 2138-2154.

30 G. Ciccarelli, S. Dorofeev, Prog. Energy Combust. Sci. 34 (2008) 499-550.

31 S. D. Watt, G. J. Sharpe, J. Fluid Mech. 522 (2005) 329-356.

32 G. J. Sharpe, M. I. Radulescu, Combust. Theory Model. 15 (2011) 691-723. 